\title{
Investigation of oscillation frequency and disorder induced dynamic phase transitions in a quenched-bond diluted Ising ferromagnet
}

\author{
Erol Vatansever, Ümit Akıncı, Yusuf Yüksel, and Hamza Polat田 \\ Department of Physics, Dokuz Eylül University, TR-35160 Izmir, Turkey
}

(Dated: September 27, 2018)

\begin{abstract}
Frequency evolutions of hysteresis loop area and hysteresis tools such as remanence and coercivity of a kinetic Ising model in the presence of quenched bond dilution are investigated in detail. The kinetic equation describing the time dependence of the magnetization is derived by means of effectivefield theory with single-site correlations. It is found that the frequency dispersions of hysteresis loop area, remanence and coercivity strongly depend on the quenched bond randomness, as well as applied field amplitude and oscillation frequency. In addition, the shape of the hysteresis curves for a wide variety of Hamiltonian parameters is studied and some interesting behaviors are found. Finally, a comparison of our observations with those of recently published studies is represented and it is shown that there exists a qualitatively good agreement.

PACS numbers: 64.60.Ht, 75.30.Kz, 75.60.-d, 75.70.Rf

Keywords: Dynamic phase transitions, Quenched bond diluted kinetic Ising model, Effective-field theory.
\end{abstract}

\section{INTRODUCTION}

When a ferromagnetic material is subject to a time dependent oscillating magnetic field, the system may not respond to the external magnetic field instantaneously, and this situation leads to some interesting phenomena. Namely, dynamic phase transitions (DPTs) and dynamic hysteresis behavior originate due to a competition between time scales of the relaxation time of the system and oscillation period of the external magnetic field. On the other hand, at high temperatures and for the high amplitudes of the periodic magnetic field, the system is able to follow the external field with some delay while this is not the case for low temperatures and small magnetic field amplitudes. This spontaneous symmetry breaking indicates the presence of a DPT [1] which shows itself in the dynamic order parameter (DOP) which is defined as the time average of the magnetization over a full period of the oscillating field. According to early experimental studies, dating back to last century, there exists an empirical law for the hysteresis loop areal scaling [2]. Up to now, a fairly well numerous theoretical studies have been dedicated to DPT and hysteresis properties of kinetic Ising models by using various methods such as Monte Carlo (MC) simulations [3-14], effectivefield theory (EFT) [15, 16] and mean-field theory (MFT) [4, 5, 17 -19, 25 29]. Apart from these, there exist a detailed geometrical description [30], and also an hysteresis criterion based on rate competition between the critical curvature and the potential-barrier height 31]. By employing standard Metropolis MC algorithm with periodic boundary conditions, the pure kinetic Ising model in a two dimensional square lattice has been simulated by Lo and Pelcovits [3], and they found some evidences of a DPT. They also investigated the behavior of dynamic

*Electronic address: hamza.polat@deu.edu.tr hysteresis for varying Hamiltonian parameters, however they did not intend to make any classification for dynamic hysteresis behavior. Afterwards, by making use of MC simulations and MFT, Acharyya and Chakrabarti [4] have presented a comprehensive investigation of the pure kinetic Ising model and they showed that the hysteresis loops are asymmetric in the dynamically ordered phase while in a dynamic disordered phase, they are symmetric around the origin in a magnetization versus field amplitude plane. In Ref. [5], the attention has been focused on the behavior of dynamic hysteresis and dynamic loop area (DLA) of a pure kinetic Ising model by using both MFT and MC calculations. In addition, the frequency variations of the coercive field have been analyzed below the zero field critical temperatures. It has been concluded that the numerical results for the dynamic coercive field show a power law frequency variation for both cases. However, the coercive field calculated by MFT becomes frequency independent in the low frequency regime. Differences between the two methods (MFT and MC) have been explained by using a kind of Landau-type double well free energy mechanism. Moreover, there exist many previously published studies such as the domain wall motion and nucleation theory 21 24 regarding the hysteresis loop mechanism [20].

On the experimental picture, it can be said that a great many of experimental studies have been devoted to DPTs and hysteresis behaviors of Co films on a $\mathrm{Cu}(001)$ surface [32], $\left[\mathrm{Co}\left(4 \mathrm{~A}^{\circ}\right) / \mathrm{Pt}\left(7 \mathrm{~A}^{\circ}\right)\right]$ multi-layer system with strong perpendicular anisotropy [33], thin polycrystalline $\mathrm{Ni}_{80} \mathrm{Fe}_{20}$ films 34], ultrathin ferromagnetic $\mathrm{Fe} / \mathrm{Au}(001)$ films [35], epitaxial $\mathrm{Fe} / \mathrm{GaAs}(001)$ thin films [36], epitaxial single ferromagnetic fcc $\mathrm{NiFe}(001)$, fcc $\mathrm{Co}(001)$, and fcc $\mathrm{NiFe} / \mathrm{Cu} / \mathrm{Co}(001)$ layers 37], $\mathrm{Fe}_{0.42} \mathrm{Zn}_{0.58} \mathrm{~F}_{2}$ [38], finemet thin films 39], and epitaxial $\mathrm{Fe} / \mathrm{GaAs}(001)$ and $\mathrm{Fe} / \operatorname{InAs}(001)$ ultrathin films [40]. After some detailed experimental investigations, it has been observed that experimental non-equilibrium dynamics of considered real magnetic systems strongly resemble the dynamic behav- 
ior predicted from theoretical calculations of a kinetic Ising model. It is clear from these works that there exists a strong evidence of qualitative consistency between theoretical and experimental studies.

Besides, the lattice models including impurities and defects have attracted considerable attention, because they are very useful to investigate the behavior of disordered systems in nature. For this purpose, more effective models have been introduced to examine the influences of disorder on the thermal and magnetic properties of real magnetic materials. For example, the effects of impurities on driving-rate-dependent energy loss in a ferromagnet under the time dependent magnetic field have been analyzed by Zheng and $\mathrm{Li}$ [41] by using several well defined models within the frameworks of MFT and MC, and they found using MFT that, the hysteresis loop area is a power law function of the linear driving rate as $A-A_{0} \propto h^{\beta}$, where, $A_{0}, h$ and $\beta$ are the static hysteresis loop area, the linear driving rate and scaling exponent of the system, respectively. Very recently, the quenched site and bond diluted kinetic Ising models under the influence of a time dependent oscillating magnetic field have been analyzed by making use of EFT [42, 43] on a two dimensional honeycomb lattice. In Ref. [42], the global phase diagrams including the reentrant phase transitions are presented by the authors for site diluted kinetic Ising model, and they showed that the coexistence regions disappear for sufficiently weak dilution of lattice sites. Following the same methodology, the authors have concentrated on the influences of quenched bond dilution process on the dynamic behavior of the system. After some detailed analysis, it has been found that the impurities in a bond diluted kinetic Ising model give rise to a number of interesting and unusual phenomena such as reentrant phenomena and the impurities to have a tendency to destruct the first-order transitions and the dynamic tricritical point [43]. Furthermore, it has also been shown that dynamically ordered phase regions get expanded with decreasing amplitude which is more evident at low frequencies.

Very recently, frequency dependencies of the dynamic phase boundary (DPB) and magnetic hysteresis of Ising model in an oscillating magnetic field have been examined by Punya et al. [19]. Based on MFT, they have represented the phase diagram in a magnetic field versus temperature plane for varying frequencies, in order to show the influence of the oscillation frequency of the external field on the hysteretic response of the system. According to their calculations, it has been found that the frequency dispersion of the DLA, the remanence and coercivity can be categorized into three distinct types for a fixed temperature. Although the detailed investigations and classifications of the hysteretic behaviors of pure kinetic Ising model have been done within the MFT [19], as far as we know, the dynamic hysteretic behavior within the EFT has not been studied for disordered kinetic Ising models. Actually, the main motivation for our work comes from the recent analysis of Ref. [19] for the investigation of hysteresis behavior and dynamic phase diagrams of kinetic Ising model. Therefore in this work, we intend to probe the effects of the quenched bond dilution process on the dynamic hysteresis behavior of kinetic Ising model in the presence of a time-dependent oscillating external magnetic field by using the EFT with correlations based on the exact Van der Waerden identity for a spin- $1 / 2$ system. Present method is quite superior to MFT, since thermal fluctuations are partly considered in EFT. The outline of the paper can be summarized as follows: The dynamic equation of motion, DOP and DLA of the quenched bond diluted kinetic Ising model have been introduced in the next section. The numerical results and related discussions are given in Section III, and finally Section IV contains our conclusions.

\section{FORMULATION}

The kinetic Ising model is given by the time dependent Hamiltonian

$$
\mathcal{H}=-\sum_{<i j>} J_{i j} S_{i}^{z} S_{j}^{z}-H(t) \sum_{i} S_{i}^{z},
$$

where the spin variables $S_{i}^{z}= \pm 1$ are defined on a honeycomb lattice $(q=3)$ and the first sum in Eq. (11) is over the nearest neighbor pairs of spins. We assume that the nearest-neighbor interactions are randomly diluted on the lattice according to the probability distribution function

$$
P\left(J_{i j}\right)=p \delta\left(J_{i j}-J\right)+(1-p) \delta\left(J_{i j}\right),
$$

where $p$ denotes the concentration of active bonds. The term $H(t)$ in Eq. (11) is a time dependent external magnetic field which is defined as

$$
H(t)=h \cos (\omega t),
$$

where $h$ and $w=2 \pi f$ represent the amplitude and the angular frequency of the oscillating field, respectively. If the system evolves according to a Glauber-type stochastic process [44] at a rate of $1 / \tau$ which represents the transitions per unit time then the dynamic equation of motion can be obtained as follows

$$
\tau \frac{d\left\langle S_{i}^{z}\right\rangle}{d t}=-\left\langle S_{i}^{z}\right\rangle+\left\langle\tanh \left[\frac{E_{i}+H(t)}{k_{B} T}\right]\right\rangle,
$$

where $E_{i}=\sum_{j} J_{i j} S_{j}$ is the local field acting on the lattice site $i$, and $k_{B}$ and $T$ denote the Boltzmann constant and temperature, respectively. If we apply the differential operator technique [45, 46] in Eq. (4) by taking into account the random configurational averages we get

$$
\frac{d m}{d t}=-m+\left.\left\langle\left\langle\prod_{j=1}^{q=3} A_{i j}+S_{j}^{z} B_{i j}\right\rangle\right\rangle_{r} f(x)\right|_{x=0},
$$

where $A_{i j}=\cosh \left(J_{i j} \nabla\right), B_{i j}=\sinh \left(J_{i j} \nabla\right)$, and $m=$ $\left\langle\left\langle S_{i}^{z}\right\rangle\right\rangle_{r}$ represents the average magnetization. $\nabla=\partial / \partial x$ 
is a differential operator, and the inner $\langle\ldots\rangle$ and the outer $\langle\ldots\rangle_{r}$ brackets represent the thermal and configurational averages, respectively. When the right-hand side of Eq. (5D) is expanded, the multispin correlation functions appear. The simplest approximation, and one of the most frequently adopted is to decouple these correlations according to

$$
\left\langle\left\langle S_{i}^{z} S_{j}^{z} \ldots S_{l}^{z}\right\rangle\right\rangle_{r} \cong\left\langle\left\langle S_{i}^{z}\right\rangle\right\rangle_{r}\left\langle\left\langle S_{j}^{z}\right\rangle\right\rangle_{r} \ldots\left\langle\left\langle S_{l}^{z}\right\rangle\right\rangle_{r},
$$

for $i \neq j \neq \ldots \neq l$ [47]. If we expand the right-hand side of Eq. (5) with the help of Eq. (6) then we obtain the following dynamic effective-field equation of motion for the magnetization of the quenched bond diluted kinetic Ising model

$$
\frac{d m}{d t}=-m+\sum_{i=0}^{q=3} \lambda_{i} m^{i},
$$

where the coefficients $\lambda_{i}$ can be easily calculated by employing the mathematical relation $\exp (\alpha \nabla) f(x)=$ $f(x+\alpha)$. Eq. (7) describes the non-equilibrium behavior of the system in the EFT formalism. Moreover, the time dependence of magnetization can be one of two types according to whether they obey the following property or not

$$
m(t)=-m(t+\pi / \omega) .
$$

A solution satisfying Eq. (8) is called symmetric solution and it corresponds to a paramagnetic or disordered phase. In this type of solution, the time dependent magnetization oscillates around zero value. The second type of solutions which does not satisfy Eq. (8) is called nonsymmetric solution which corresponds to ferromagnetic (i.e. ordered) phase where the time dependent magnetization oscillates around a non-zero value.

The time averaged magnetization over a full cycle of the oscillating magnetic field acts as DOP which is defined as follows [17]

$$
Q=\frac{\omega}{2 \pi} \oint m(t) d t
$$

where $m(t)$ is a stable and periodic function. On the other hand, the DLA corresponding to energy loss due to the hysteresis is defined as [6]

$$
A=-\oint m(t) d H=h \omega \oint m(t) \sin (\omega t) d t .
$$

The behavior of DOP and DLA as a function of the temperature for selected Hamiltonian parameters can be obtained by solving Eqs. (7), (91) and (10) numerically. The remanent magnetization and coercivity can be calculated by benefiting from hysteresis loop $(m(t)-H(t)$ curve) in order to understand and clarify the behavior of the quenched bond diluted system.

\section{RESULTS AND DISCUSSION}

In this section, in order to understand the dynamic evolution of the magnetic system in detail, we will focus our attention on the hysteretic response and DPT properties of the quenched-bond diluted kinetic Ising model under a time-dependent applied magnetic field. It is well known that depending on the value of the applied field frequency, the magnetic system can exist in a dynamically ordered or disordered phase. From this point of view, we give the DPB in a $\left(k_{B} T_{c} / J-\omega\right)$ plane with four selected amplitudes of the applied field ( $h=0.25,0.5,0.75,1.0)$, and for some selected values of the active bond concentration in Figs. 1(a-d), in order to clarify the influence of applied field frequency on the DPT properties. At first sight, one can easily observe from Figs. 1(a-d) that the dynamically ordered phases get expanded with increasing applied field frequency for all considered active bond concentrations. Actually, this is an expected result since as the frequency increases then the time-dependent magnetization can not follow applied magnetic field immediately, hence the dynamically ordered phase region moves upward with the increasing frequency. We can also mention that aforementioned situations are also reported in previously published works 1, 16, 17, 19, 42, 43].

On the other hand, as the value of $p$ decreases, starting from pure case (i.e. $p=1.0$ ), the magnetic system exhibits unusual and interesting behaviors. For instance, dynamically disordered phases occur in the low temperature regions and also reentrant behavior emerges. Another important consequence of decreasing $p$ is that the dynamically ordered phases get narrower for all values of the considered applied field amplitudes. These observations can also be explained by the following mechanism: The energy contribution which comes from the spin-spin interaction gets smaller when the value of $p$ is decreased. So, the system can undergo a DPT at a lower critical temperature, since the energy contribution originating from the temperature and (or) magnetic field overcomes the ferromagnetic spin-spin interactions. As a result of this mechanism, the dynamically ordered phase regions in $\left(k_{B} T_{c} / J-\omega\right)$ plane get narrower. As discussed above, the existence or absence of the reentrant behavior strongly depends on the applied field amplitude, as well as oscillation frequency. As an example, let us consider the curves with $p=0.85$ to show the influences of the applied field amplitude on the DPB, for selected values of $h$. As seen in Figs. 1(a-d), for $h=0.25$ and 0.5, the magnetic system exhibits reentrant behavior whereas this behavior disappears with further increase in $h$, such as for $h=0.75$ and 1.0 .

It is known that applied field frequencies naturally affect the dynamic evolution of a magnetic system. In order to show the influences of the applied field frequencies on the system, the temperature dependencies of the DOP $(Q)$ and DLA $(A)$ are depicted in Figs. 2(a-d) for values of $p=0.85$ and $h=0.25$ corresponding to Fig. 1(a). 

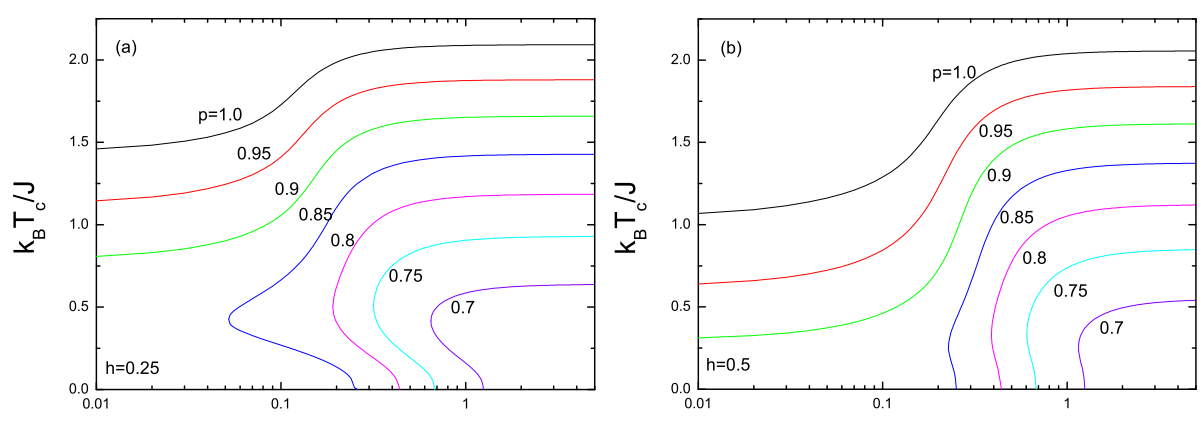

$\omega$
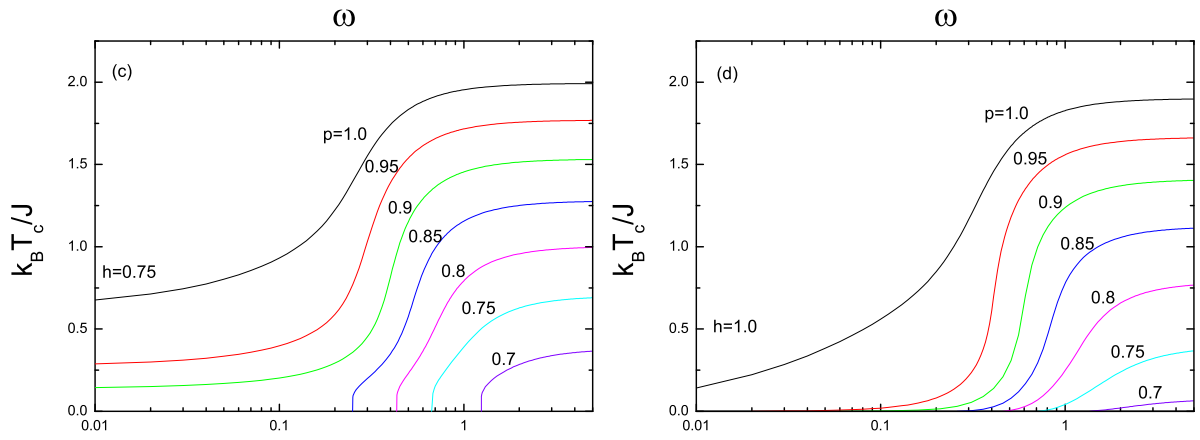

$\omega$

$\omega$

FIG. 1: Dynamic phase boundaries of the quenched bond diluted kinetic Ising system in a $\left(k_{B} T_{c} / J-\omega\right)$ plane with some selected values of amplitude of the applied magnetic field. (a) $h=0.25$, (b) $h=0.50$, (c) $h=0.75$ and (d) $h=1.0$. The numbers accompanying each curve denote the value of active bond concentrations.

In accordance with this purpose, we separated the frequency space into four regions: The order of the magnetic system is dynamically paramagnetic in first region for $\omega=0.01$. One can see from Fig. 2(a) that the DLA gradually increases and exhibits a local maxima with a Schottky-like rounded hump and then tends to diminish with increasing temperature. On the other side, it is clear from Fig. 2(b) that there exists two successive second order DPTs for a selected frequency value of $\omega=0.1$, and this type of treatment corresponds to second region. The first DPT is from paramagnetic to ferromagnetic phase while the second DPT is from ferromagnetic to paramagnetic phase. For the same Hamiltonian parameters, the DLA exhibits two local maxima and two whiskers and the positions of the whiskers may correspond to DPT points. In Fig. 2(c), we represent the thermal variation of the DOP and DLA curves for $\omega=0.5$ which indicates the third region. It can be easily seen from Fig. 2(c) that only one DPT emerges in magnetic system for $\omega=0.5$. DOP curve for $\omega=0.5$ increases form its ground state value with increasing temperature which originates from thermal agitations and it decreases with further increasing temperatures and then falls to zero at a second order DPT temperature. Similarly, with the increasing temperature, starting from zero, the DLA decreases and shows a local minima at the dynamically ordered phase region and then it exhibits a sharp peak near the DPT point. In the following analysis, let us consider the higher value of the applied field frequency such as $\omega=10.0$ which refers to fourth region. As we can see from Fig. 2(d) that the magnetic system exhibits a second order DPT. However, we should also mention that the value of phase transition temperature observed for $\omega=10.0$ is slightly higher than that of $\omega=0.5$. Actually, this is an expected result because an increase in the applied field frequency makes the occurrence of phase transition difficult, hence the dynamically ordered phase gets expanded with the increasing applied field frequency. For given Hamiltonian parameters, the DLA shows a peak above the second order DPT point. We should note that the existence of a peak on the DLA near DPT point has also been indicated in previous investigations for various models [6, 12, 15, 16, 48].

We give the frequency dispersion curves of the DLA in Figs. 3(a-d) for four selected $p$ values with a variety of $h$ values. We should note that the reduced temperature in this study is fixed as $k_{B} T / J=1.0$ which is lower than the transition temperature $T_{c}$ of a pure system with EFT for $q=3$ in the absence of external field. This selection of the temperature allows the system to undergo an amplitude, frequency or disorder induced dynamic phase transition. Fig 3(a) is plotted for $p=1.0$ where all ferromagnetic spin-spin exchange interactions $(J>0)$ are active. At first sight, one can clearly observe in Fig. [3(a) that DLA curves can exhibit two types of characteristic behavior. The first one corresponds to a dynamically ordered phase where the DLA increases as the applied field frequency increases and then exhibits a characteristic rounded peak (or local maxima) for the relatively low field amplitude values such as $h=0.1,0.2,0.3,0.4$ and 0.5. These curves are identical to type-I curves of 

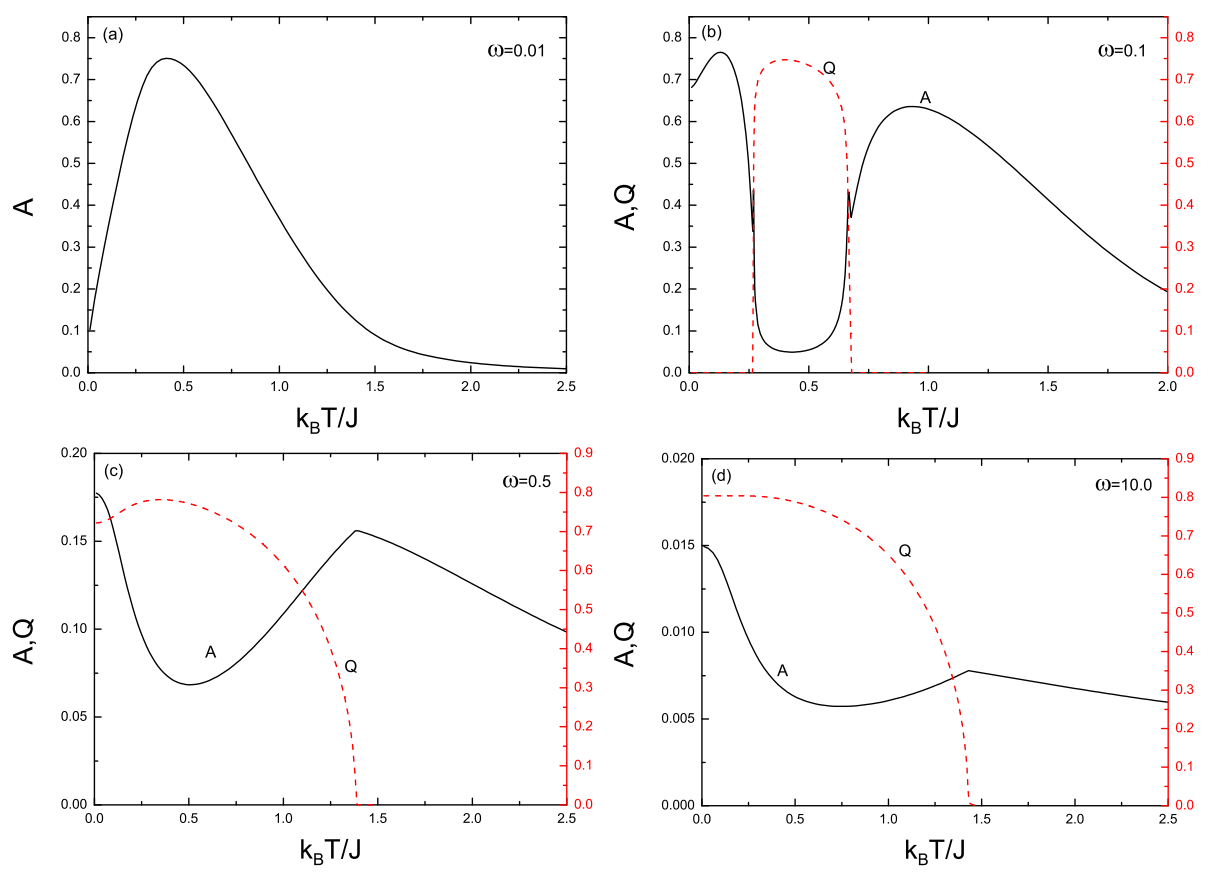

FIG. 2: Temperature variations of the dynamic order parameter and dynamic loop area for considered values of the $h(=0.25)$ and $p(=0.85)$.

Ref. [19]. As is expected, the DLA is relatively close to zero at the low and high applied field frequency regions in the dynamically ordered phases. On the other hand, for $h=0.6,0.7,0.8,0.9$ and 1.0 values, DLA curves start from a nonzero value and increases with increasing frequency values then exhibit a finite cusp at a second order phase transition temperature at which the shape of hysteresis loop changes from symmetric to asymmetric and also a rounded hump in high frequency regime which corresponds to second type of characteristic behavior. These curves correspond to type-II curves in Ref. [19]. Finite cusps are found to be discontinuous for low $h$ and weak disorder. The rounded humps observed in DLA curves in high frequency regime disappears for increasing $h$ and (or) decreasing $p$ values. For instance, for $h=0.6$ with $p=1.0$, the DLA exhibits a discontinuous cusp and a rounded distinct hump while it displays only a continuous cusp for $h=1.0$ and $p=1.0$. We can mention that the characteristic cusp of $(h, p)=(0.6,1.0)$ curve arises from the frequency evolution of symmetric hysteresis loop, whereas the rounded hump of this curve originates from the frequency evolution of asymmetric hysteresis loop. On the other side, it is known that the phase transition temperature decreases with increasing amount of disorder. The effects of the aforementioned situation on the frequency dispersion can be seen in Figs. 3(b-d) which have been plotted for $p=0.9,0.8$, and 0.7. One can observe in Figs. 3(b-d) that as the disorder effects become dominant in the system then the representative type-I curves tend to evolve into type-II, and type-II curves have a tendency to turn into type-III curves defined in Ref. [19] which represent the paramagnetic DLA dispersion curves. In contrast to type-I curves, type-III curves have a finite residual value in the limit of $\omega \rightarrow 0$. In addition, the type-III curves are characterized by a rounded hump, as type-I curves. Based on these observations, we see that DLA dispersion curves clearly reveal the signs of the oscillation frequency and disorder induced dynamic phase transitions in the present system.

In Figs. 4(a-d), we present the frequency dependence of remanent magnetization curves corresponding to DLA curves in Fig. 3. Here we select four typical values of disorder parameter $p$ with a variety of field amplitude $h$ values. Remanent magnetization is defined as the residual magnetization of a ferromagnetic material after an external magnetic field is removed. Hence the remanence data can be easily extracted from calculated hysteresis loops. At first glance, it can be easily seen that depending on amplitude and frequency of the external magnetic field, as well as the amount of disorder, remanence curves can exhibit three distinct characteristics corresponding to type-I, type-II and type-III profiles of DLA curves. Corresponding to type-I DLA curves, either branches of remanent magnetization curves are positively valued which means that the system is ordered ferromagnetically in the whole range of oscillation frequency $\omega$. Increasing the amount of disorder or amplitude of the external field, representative type-I remanence curves may evolve to typeII and type-III curves in order. Type-II remanence curves exhibit equally valued branches with opposite signs in the low frequency region indicating that the system is in a dynamically paramagnetic state. As the frequency increases then the negative branch of a representative typeII remanence curve changes its sign at a critical value of 

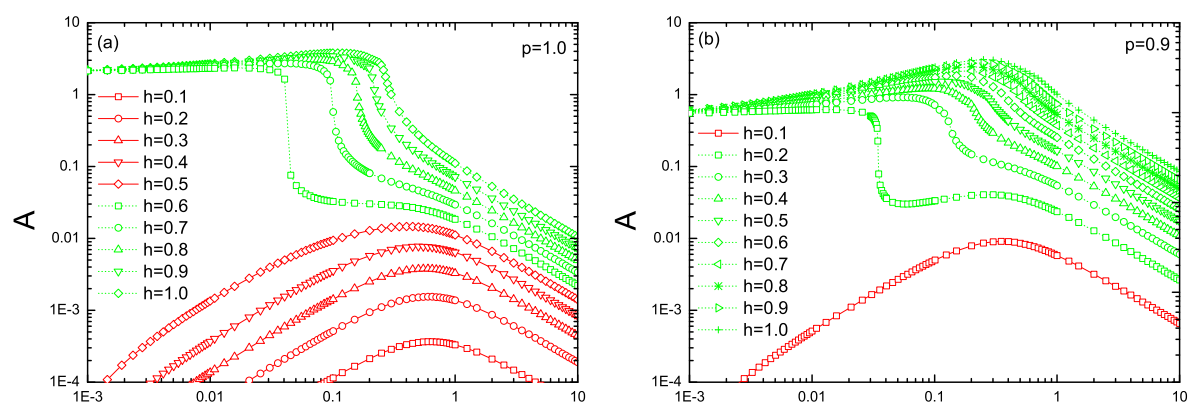

$\omega$

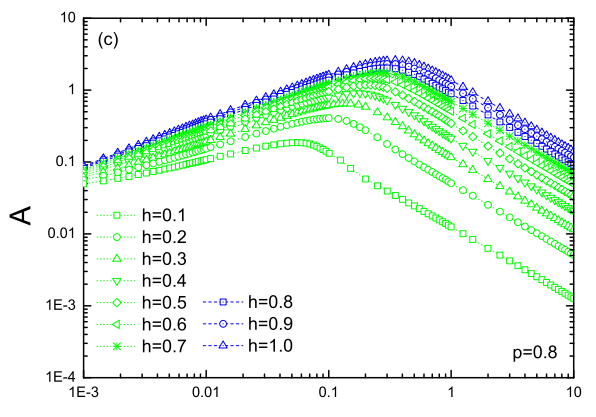

$\omega$

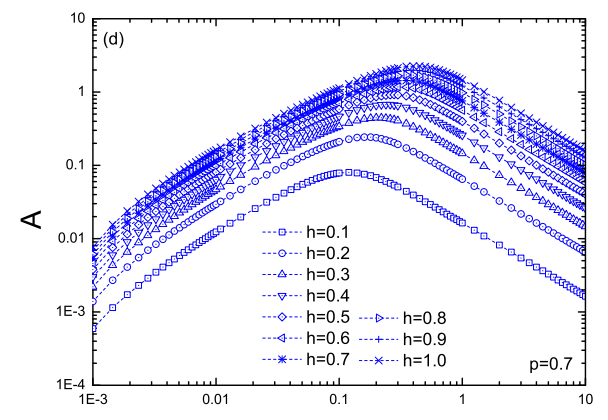

$\omega$

FIG. 3: The frequency dispersion of the hysteresis loop area curves with considered applied field amplitudes for selected values of active bond concentrations (a) $p=1.0$, (b) $p=0.9$, (c) $p=0.8$ and (d) $p=0.7$.

frequency, hence we understand that the corresponding hysteresis loop loses its symmetry and becomes dynamically ordered. Hence this behavior can be considered as a frequency induced phase transition. For sufficiently high amplitudes and (or) for a sufficiently large amount of disorder, the observed type-II curves evolve to typeIII characteristics. In this case, either branches of remanence curves are symmetric with opposite signs which indicates that the system is dynamically disordered for the whole range of oscillation frequency spectrum. Remanent magnetization is a vital property for materials used in magnetic information storage devices. Namely, in case of some fixed values of external field amplitude and oscillation frequency, as well as an amount of existing disorder, for a device made of a material with higher remanence property there will be less chance that the information stored in the media will be erased due to some external sources [4]. From this point of view, it is important to note that according to Fig. 4(d), there exists an optimum field frequency for a disordered material under an oscillating magnetic field at which the type-III remanence (i.e. the distance between positive and negative remanent magnetizations) exhibit a maximum value, and as the field amplitude increases then the optimum frequency value shifts towards higher frequency values.

As another characteristic feature of hysteresis loops, we have examined the coercivity of the system which is defined as the minimum required reversal magnetic field, in order to reduce the remanent magnetization to zero. For storage media, a material with a high coercivity is desired to preserve the stored data from unwanted external factors. We represent the variation of the coercive field curves as a function of oscillation frequency in Figs. 5)(a-d) for some selected values of disorder parameter $p$ and external field amplitude $h$. For the DLA curves with type-I characteristics, there is no coercivity for any field amplitude, oscillation frequency, or disorder, because all hysteresis curves corresponding to type-I behavior have asymmetric shape. This means that the instantaneous magnetization can not follow the external magnetic field. For type-II characteristics however, we see that at low frequencies, absolute values of either branches in a coercivity curve of the system increase for a while then tend to decrease with further increasing frequencies. After a critical frequency value, these branches lose their symmetric shape then coercive fields disappear and a dynamic phase transition from a dynamic paramagnetic phase to a ferromagnetic one takes place. We note that nonsymmetric coercivity data have not been shown in Fig. 5. We again see that an oscillation frequency induced dynamic phase transition takes place in representative type-II curves. If the magnetic field amplitude is large enough or if there exists a sufficiently strong disorder (see Figs. 5 , and d), coercivity curves may evolve from type-II to type-III characteristics. Since the type-III hysteresis curves are symmetric, coercive field can be observed for the whole range of oscillation frequency values. However, for typeIII behavior, coercivity is rather small at low frequencies. As the field frequency increases then coercivity of the system increases and in the limit of $\omega \rightarrow \infty$ it saturates to its maximum value. The maximum value of coercivity 

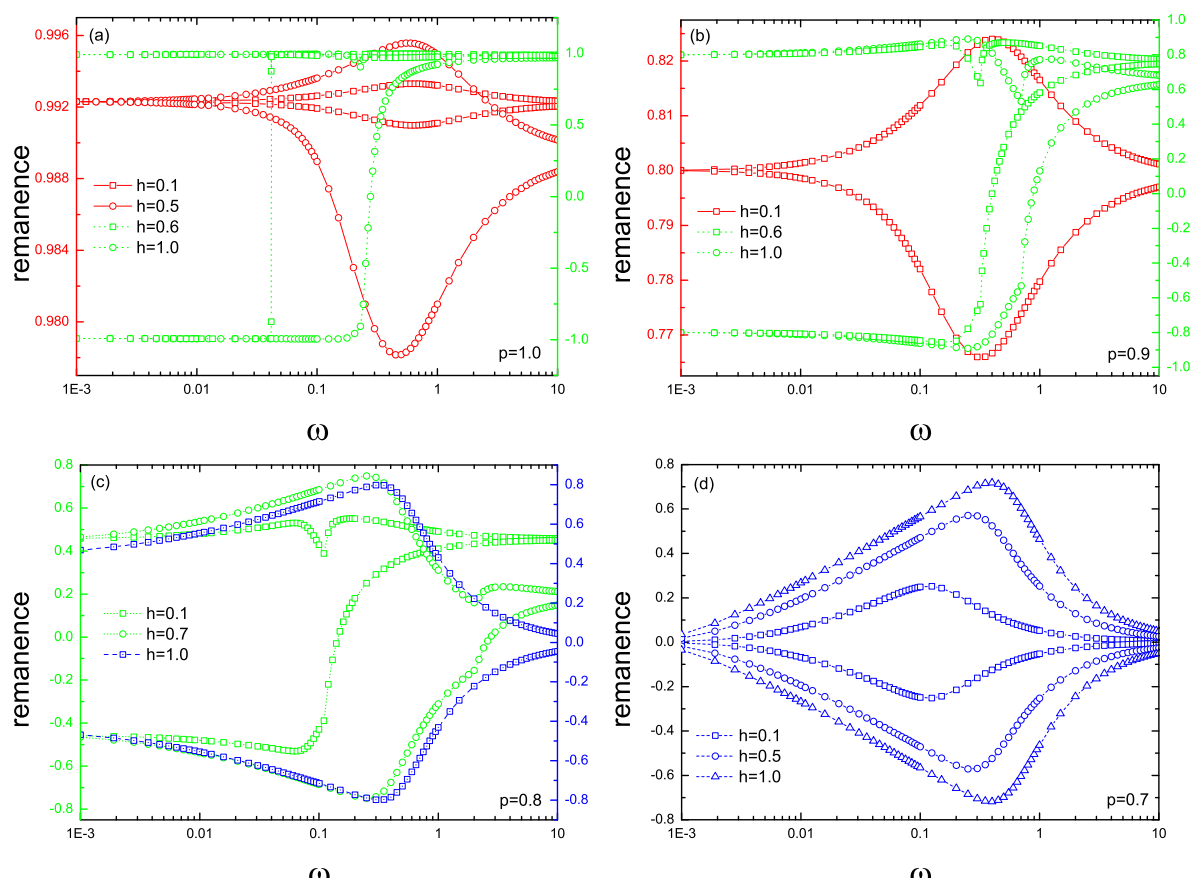

$\omega$

FIG. 4: Frequency dispersion of remanent magnetization for a variety of applied field amplitudes with selected active bond concentrations (a) $p=1.0$, (b) $p=0.9$, (c) $p=0.8$ and (d) $p=0.7$.

is noting but just the applied amplitude of the external magnetic field for sufficiently high frequencies. Hence, we can conclude that the greater amplitude of the external magnetic field, we get more enhanced coercivity.

The two fundamental tools, namely coercivity and remanence properties allow us to make predictions on the shape of the hysteresis loops. Effect of the field frequency on the shape of typical hysteresis loops corresponding to Figs. 34 4, and 5 are depicted in Fig. 6 for a wide variety of oscillation frequencies within the range $0.01 \leq \omega \leq 10.0$ with given values of $p$ and $h$. In Fig. 6(a), we present the hysteresis loop curves corresponding to type-I characteristics of DLA curves. As seen in Fig. 6(a), hysteresis curves exhibit positive valued narrow loops at low frequencies then evolve into a horizontal line with increasing frequencies. Type-II curves are shown in Fig. [6(b), where the low frequency paramagnetic loops turn into high frequency ferromagnetic loops due to a frequency induced dynamic symmetry loss in the system. Moreover, according to Fig. 6(c), symmetric type-III hysteresis curves keep their dynamic paramagnetic state and exhibit remanence and coercivity with branches having both positive and negative values. We can conclude from Fig. 6(c) that low frequency thin and $S$-shaped hysteresis loops evolves into broad squarelike shapes then turn into elliptical loops for sufficiently high frequencies. If we increase the frequency further, these elliptical loops become horizontal lines. Related to the frequency evolutions of the hysteresis curves, we should also note that a qualitative consistency is found between our numerical findings and experimental obser- vations $[32,35,50]$.

In the following analysis, let us focus on the influence of disorder on the frequency dispersion of hysteretic properties of the system. In Figs. 7(a) and 7(b), we represent variation of coercivity and remanence curves as a function of oscillation frequency for some selected values of disorder parameter $p$ with a given field amplitude $h=0.5$. From Fig. Z(a), we see that as the concentration of diluted bonds increases then the zero frequency branches of coercivity curves vanish as $\omega \rightarrow 0$. In type-II curves, critical frequency at which the system exhibit a dynamic phase transition increases with increasing disorder. This frequency value can be regarded as a cut-off frequency at which the symmetric loops turn into asymmetric loops. Hence, the non-symmetric coercivity data has not been considered in Fig. 7(a). Moreover, coercivity curves exhibiting type-II characteristics may evolve into type-III behavior, if the concentration of diluted bonds exceeds a critical value (i.e. percolation threshold). Hence, we understand that the system exhibits a disorder induced dynamic phase transition. In Fig. 7(b), we illustrate the dependence of frequency dispersion of remanence curves on the concentration of disordered bonds with a fixed external field amplitude $h=0.5$ corresponding to Fig. 7(a). Type-I curves in Fig. 7(b) exhibit a positive remanence which indicates that the system stays in a dynamic ferromagnetic order for the whole range of frequency spectrum. By increasing disorder, type-I curves turn into type-II characteristics where we can observe that either branches of remanence curves tend to lose their symmetric shape with increasing frequency which 

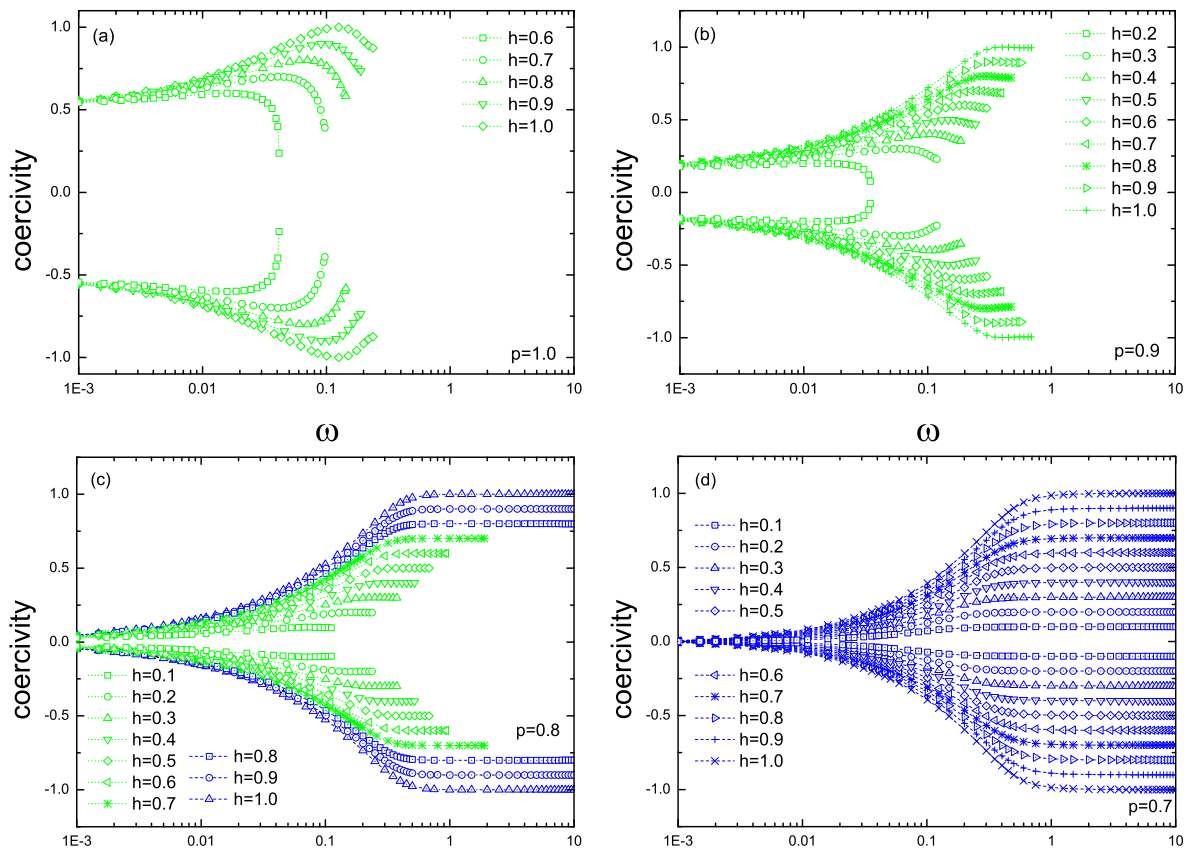

$\omega$

$\omega$

FIG. 5: Frequency dispersion of the coercivity for a variety of applied field amplitudes with selected values of active bond concentrations (a) $p=1.0$, (b) $p=0.9$, (c) $p=0.8$ and (d) $p=0.7$.

signals the appearance of a frequency induced dynamic phase transition. In a similar manner, we observe in Fig. 7(b) that for sufficiently low concentration of magnetic bonds, the remanent magnetization curves may exhibit type-III characteristics where the system always exhibit a dynamically disordered phase for the whole range of oscillation frequency spectrum.

As a final investigation, we discuss the evolution of some typical hysteresis loops corresponding to remanence and coercivity properties depicted in Fig. 7 In Fig. 8 we fix the amplitude of the external magnetic field as $h=0.5$. Fig. 8 (a) corresponds to low frequency situation $(\omega=0.01)$. From Fig. 8 a, we see that a disorder induced dynamic phase transition takes place in the system with increasing disorder. Hysteresis loops exhibit a positive valued horizontal line in the pure case whereas as the amount of disorder increases then the loop area gets narrower and we observe S-shaped thin loops. On the other hand, the situation is depicted for relatively high frequency values such as $\omega=1.0$ in Fig. 8 (b). In this case, asymmetric loops corresponding to ferromagnetic order become elliptical symmetric loops in the dynamic paramagnetic phase with increasing disorder. Hence, we can conclude that influence of disorder on the shape of hysteresis loops strictly depends on the magnitude of the applied field frequency. Aforementioned results are also consistent with the observations illustrated in Fig. 7.

\section{CONCLUSIONS}

In conclusion, by using EFT with correlations, we have investigated frequency dispersion features of dynamic loop area, coercivity and remanent magnetization of a kinetic Ising model in the presence of quencheddiluted bonds. First of all, in order to gain some general insight regarding the influence of bond disorder, external field amplitude, and oscillation frequency on the dynamic phase transition properties of the system we have demonstrated the phase diagrams of our model in a $\left(k_{B} T_{c} / J-\omega\right)$ plane for weak $(h=0.25)$, intermediate $(h=0.5,0.75)$ and large $(h=1.0)$ values of the external field amplitude with some selected values of disorder parameter $p$. We have found that critical frontiers between symmetric and asymmetric phases in $\left(k_{B} T_{c} / J-\omega\right)$ plane are strongly dependent on amplitude and frequency of oscillating field, as well as amount of disorder.

EFT is superior to conventional MFT predictions owing to the fact that the single spin correlations, in other words thermal fluctuations are partially considered. Hence, we take the recently published work performed by Ref. [19] one step forward by considering single-site correlations between different lattice sites, and by considering the presence of disordered bonds. However, it is worth to note that the approximation method used in the present work, does not take into account the local structure of the lattice. In other words, EFT cannot distinguish between a two dimensional triangular lattice and a three dimensional simple cubic lattice, since both have a coordination number $q=6$. In this context, the results obtained in 

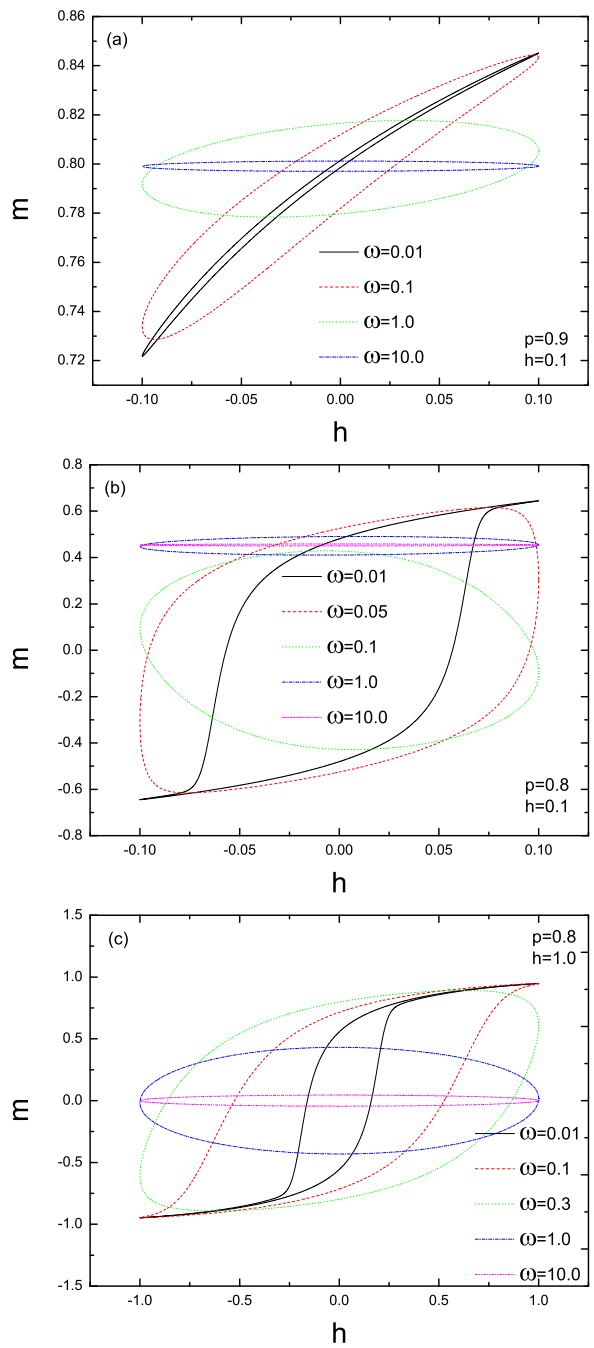

FIG. 6: Hysteresis loops of the quenched bond diluted kinetic Ising system for several values of applied field frequencies and amplitudes with some selected values of active bond concentrations.

the present paper depend on the coordination number of the system.

The results obtained in the present paper qualitatively support the recent findings based on MFT [19] for the pure case. Namely, we have observed that frequency dispersion of dynamic loop area (DLA) can be classified into three distinct types. In type-I behavior, the system always exhibits asymmetric hysteresis loops at any oscillation frequency for small field amplitudes and weak amount of disorder whereas type-II curves are characterized by a frequency induced phase transition where the low frequency symmetric loops become asymmetric loops with increasing oscillation frequency. On the other hand, type-III behavior is essentially represented by symmetric hysteresis loops for the whole range of frequency spectrum when the external field amplitude is sufficiently large and (or) if there exists sufficiently strong dilution of ferromagnetic nearest-neighbor bonds. Therefore we
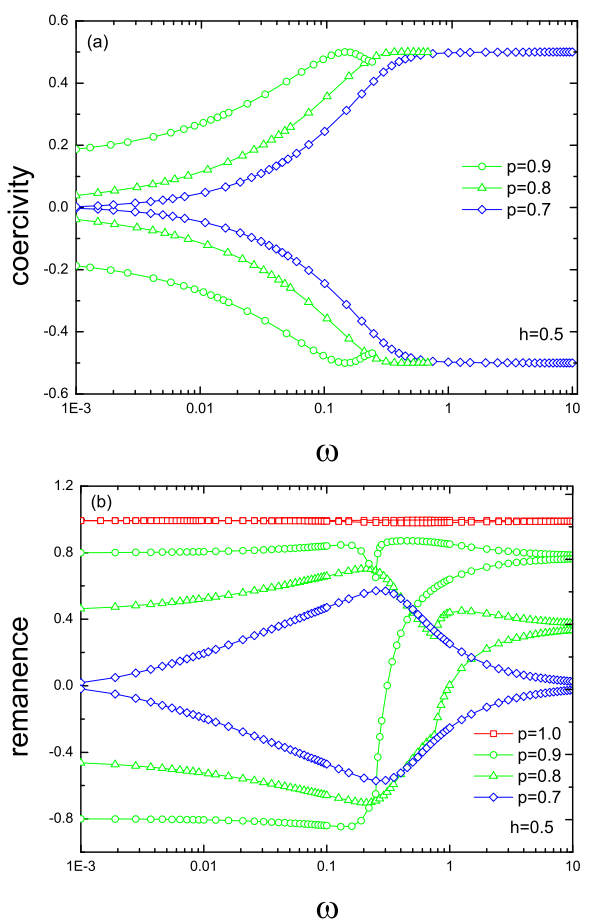

FIG. 7: Variation of the coercive field (a) and the remanent magnetization (b) as a function of the applied field frequency for a field amplitude value of $h=0.5$ with some selected values of disorder parameter $p$.
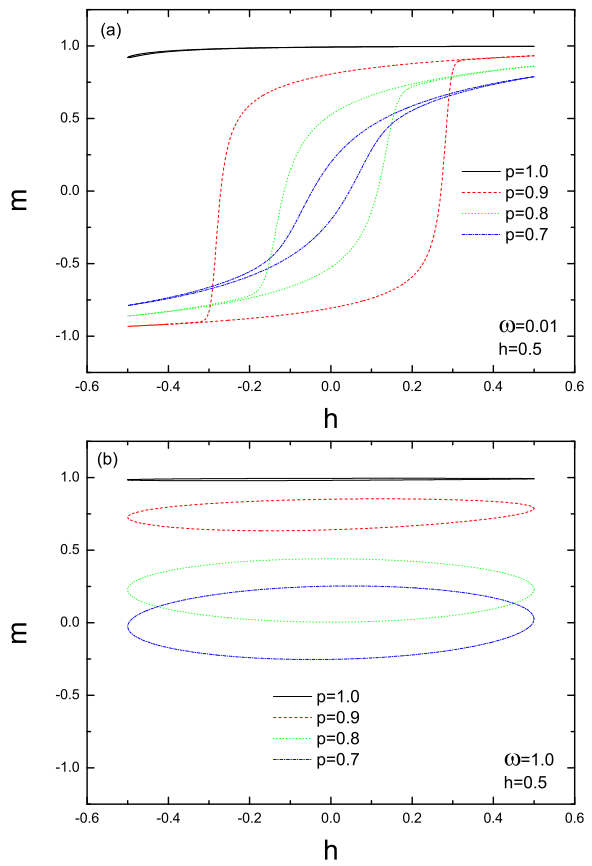

FIG. 8: Hysteresis curves of the quenched bond diluted kinetic Ising system for several values of active bond concentrations with selected values of applied field frequencies and amplitude. 
can conclude that type-II curves exhibit frequency induced dynamic phase transitions whereas evolution of DLA curves from type-I to type-III characteristics is due to a disorder induced effect or as a consequence of large amplitudes of the external magnetic field.

Aforementioned observations have also been supported by detailed investigation of frequency dispersions of coercivity and remanent magnetizations. From the technological point of view, in magnetic information storage devices a material with high coercivity and large remanence is desired to preserve the stored data from unwanted external factors. In this regard we have found that there exists an optimum field frequency for a disordered material under an oscillating magnetic field at which the type-III remanence (i.e. the distance between positive and negative remanent magnetizations corresponding to type-III) exhibit a maximum value, and as the field amplitude increases then the optimum frequency value shifts towards higher frequency values. Finally, we have ob- served that influence of disorder on the shape of hysteresis loops strictly depends on the magnitude of the applied field frequency.

As a conclusion, we hope that the results obtained in this work would shed light on the further investigations of the dynamic nature of the critical phenomena in disordered systems and would be beneficial from both theoretical and experimental points of view.

\section{Acknowledgements}

The authors (E.V. and Y.Y.) would like to thank the Scientific and Technological Research Council of Turkey (TÜBITAK) for partial financial support. The numerical calculations reported in this paper were performed at TÜBITAK ULAKBIM (Turkish agency), High Performance and Grid Computing Center (TRUBA Resources).
[1] B.K. Chakrabarti, and M. Acharyya, Rev. Mod. Phys. 71, 847 (1999), and the references therein.

[2] C.P. Steinmetz, Trans. Am. Inst. Electr. Eng. 9, 3 (1892).

[3] W.S. Lo, and R.A. Pelcovits, Phys. Rev. A 42, 7471 (1990).

[4] M. Acharyya, and B.K. Chakrabarti, Phys. Rev. B 52, 6550 (1995).

[5] M. Acharyya, Physica A 253, 199 (1998).

[6] M. Acharyya, Phys. Rev. E 58, 179 (1998).

[7] M. Rao, H.R. Krishnamurthy, and R. Pandit, Phys. Rev. B 42, 856 (1990).

[8] S.W. Sides, P.A. Rikvold, and M.A. Novotny, Phys. Rev. E 59, 2710 (1999).

[9] S.W. Sides, P.A. Rikvold, and M.A. Novotny, Phys. Rev. Lett. 81, 834 (1998).

[10] S.W. Sides, P.A. Rikvold, and M.A. Novotny, Phys. Rev. E 57, 6512 (1998).

[11] G. Korniss, P.A. Rikvold, and M.A. Novotny, Phys. Rev. E 66, 056127 (2002).

[12] H. Zhu, S. Dong, and J.-M. Liu, Phys. Rev. B 70, 132403 (2004).

[13] F. Zhong, Phys. Rev. B 66, 060401(R) (2002).

[14] M. Acharyya, Phys. Rev. E 59, 218 (1999).

[15] X. Shi, G. Wei, and L. Li, Phys. Lett. A 372, 15922 (2008).

[16] B. Deviren, O. Canko, and M. Keskin, Chin. Phys. B 19, 050518 (2010).

[17] T. Tomè, and M.J. de Oliveira, Phys. Rev. A 41, 4251 (1990).

[18] M. Acharyya, J. Phys. A: Math. Gen. 27, 1533 (1994).

[19] A. Punya, R. Yimnirun, P. Laoratanakul, and Y. Laosiritaworn, Physica B 405, 3488 (2010).

[20] I.F. Lyuksyutov, T. Nattermann, and V. Pokrovsky, Phys. Rev. B 59, 4260 (1999).

[21] T. Nattermann, V. Pokrovsky, and V.M. Vinokur, Phys. Rev. Lett. 87, 197005 (2001).

[22] T. Nattermann, and V. Pokrovsky, Physica A 340, 625 (2004).

[23] A. Misra, and K. Chakrabarti, Europhys. Lett. 52, 311
(2000).

[24] F. Schütze, and T. Nattermann, Phys. Rev. B, 83024412 (2011).

[25] L. Wang, B.H. Teng, Y.H. Rong, Y. Lü, and Z.C. Wang, Sol. State Comm. (2012) http://dx.doi.org/10.1016/j.ssc.2012.04.076

[26] M.F. Zimmer, Phys. Rev. E 47, 3950 (1993).

[27] C.N. Luse, A. Zangwill, Phys. Rev. E 50, 224 (1994).

[28] J.-M. Liu, H.L.W. Chan, C.L. Choy, and C.K. Ong, Phys. Rev. B 65, 014416 (2001).

[29] O.S. Sariyer, A. Kabakcioglu, and A.N. Berker, arXiv.1206.0230v1

[30] I.D. Mayergoyz, Mathematical Model of Hysteresis, Springer-Verlag, Berlin, (1991).

[31] R. Gilmore, Phys. Rev. A 20, 2510 (1979).

[32] Q. Jiang, H.-N. Yang, and G.-C. Wang, Phys. Rev. B 52, 14911 (1995).

[33] D.T. Robb, Y.H. Xu, O. Hellwig, J. McCord, A. Berger, M.A. Novotny, and P.A. Rikvold, Phys. Rev. B 78, $134422(2008)$.

[34] B.C. Choi, W.Y. Lee, A. Samad, and J.A.C. Bland Phys. Rev. B 60, 11906 (1999).

[35] Y.-L. He, and G.-C. Wang, Phys. Rev. Lett. 70, 2336 (1993).

[36] W.Y. Lee, B.-Ch. Choi, J. Lee, C.C. Yao, Y.B. Xu, D.G. Hasko, and J.A.C. Bland, Appl. Phys. Lett. 74, 1609 (1999).

[37] W.Y. Lee, B.-Ch. Choi, Y. B. Xu, and J.A.C. Bland, Phys. Rev. B 60, 10216 (1999).

[38] A.R.-Rivera, J.M. Ferreira, and F.C. Montenegro, J. Magn. Magn. Mat. 226-230, 1309 (2001).

[39] L. Santi, R.L. Sommer, A. Magni, G. Durin, F. Colaiori, and S. Zapperi, IEEE Trans. Magn. 39, 2666 (2003).

[40] T.A. Moore, J. Rothman, Y.B. Xu, and J.A.C. Blanda, J. Appl. Phys. 89, 7018 (2001).

[41] G.-P. Zheng, and M. Li, Phys. Rev. B 66, 054406 (2002).

[42] U. Akinci, Y. Yüksel, E. Vatansever, and H. Polat, Physica A (2012), doi: 10.1016/j.physa.2012.06.060.

[43] E. Vatansever, B.O. Aktas, Y. Yüksel, U. Akinci, and H. 
Polat, J. Stat. Phys. 147, 1068 (2012).

[44] R.J. Glauber, J. Math. Phys. 4, 294 (1963).

[45] R. Honmura, and T. Kaneyoshi, J. Phys. C 12, 3979 (1979).

[46] T. Kaneyoshi, Acta Phys. Pol. A 83, 703 (1993).

[47] I. Tamura, and T. Kaneyoshi, Prog. Theor. Phys. 66, 1892 (1981).

[48] Z. Huang, Z. Chen, F. Zhang, and Y. Du, Phys. Lett. A
338, 485 (2005).

[49] C. Altavilla, E. Ciliberto, Inorganic Nanoparticles: Synthesis, Applicaions, and Prespectives, Taylor\&Francis, CRC Press, p33 (2010).

[50] J.-M. Liu, Q. Xiao, Z.G. Liu, H.L.W. Chan, and N.B. Ming, Mat. Chem. Phys. 82, 733 (2003). 